\title{
Selenium status in neonates with connatal infection
}

\author{
Lennart Wiehe ${ }^{1} \dagger$, Malte Cremer $^{2} \dagger$, Monika Wisniewska ${ }^{1} \dagger$, Niels-Peter Becker ${ }^{1}$, Eddy Rijntjes ${ }^{1}$, \\ Janine Martitz ${ }^{1}$, Sandra Hybsier ${ }^{1}$, Kostja Renko ${ }^{1}$, Christoph Bührer ${ }^{2}$ and Lutz Schomburg ${ }^{1 *}$ \\ ${ }^{1}$ Institute for Experimental Endocrinology, Charité Universitätsmedizin Berlin, Suedring 10, Campus Virchow-Klinikum, \\ D-13353 Berlin, Germany \\ ${ }^{2}$ Department of Neonatology, Charité Universitätsmedizin Berlin, Mittelallee 9, Campus Virchow-Klinikum, D-13353 Berlin, \\ Germany
}

(Submitted 14 February 2016 - Final revision received 25 April 2016 - Accepted 9 May 2016 - First published online 8 June 2016)

\section{Abstract}

Infectious diseases impair Se metabolism, and low Se status is associated with mortality risk in adults with critical disease. The Se status of neonates is poorly characterised, and a potential impact of connatal infection is unknown. We hypothesised that an infection negatively affects the Se status of neonates. We conducted an observational case-control study at three intensive care units at the Charité-Universitätsmedizin Berlin, Germany. Plasma samples were collected from forty-four neonates. On the basis of clinical signs for bacterial infection and concentrations of IL- 6 or C-reactive protein, neonates were classified into control ( $n$ 23) and infected ( $n$ 21) groups. Plasma Se and selenoprotein P (SePP) concentrations were determined by X-ray fluorescence and ELISA, respectively, at day of birth (day 1) and $48 \mathrm{~h}$ later (day 3). Se and SePP showed a positive correlation in both groups of neonates. Se concentrations indicative of Se deficit in adults $(<20 \mu \mathrm{g} / \mathrm{l})$ were observed in four infected neonates and one control subject, and three infected neonates had very low SePP concentrations $(<0 \cdot 5 \mathrm{mg} / \mathrm{l})$. The univariate analysis revealed a significant difference in Se and SePP concentrations between the groups. Both parameters correlated inversely to IL-6 in neonates with severe inflammation (IL-6 > $500 \mathrm{ng} / \mathrm{l}$ ). During antibiotic therapy, SePP increased significantly from day 1 ( $1 \cdot 03$ (sD $0 \cdot 10) \mathrm{mg} / \mathrm{l}$ ) to day $3(1.34$ (sD 0.10) $\mathrm{mg} / \mathrm{l})$, indicative of improved hepatic Se metabolism. We conclude that both Se and SePP are suitable biomarkers for assessing Se status in neonates and for identifying subjects at risk of deficiency.

\section{Key words: Selenoproteins: Gentamicin: Intensive care: IL-6: Micronutrients}

Se is an important trace element and micronutrient, essentially needed for the biosynthesis of selenocysteine-containing selenoproteins. In humans, the selenoproteome comprises enzymes implicated in redox control, antioxidative defence, thyroid hormone metabolism, endoplasmic reticulum function and other important biochemical pathways including the systemic Se transport by selenoprotein P (SePP) ${ }^{(1)}$. A few cases of inherited defects in selenoprotein biosynthesis have been characterised. Symptoms may include severe muscular dystrophy, cognitive impairment, endocrine alterations and sensitivity towards oxidative noxae ${ }^{(2)}$. On a population-wide scale, low Se intake and suboptimal selenoprotein expression have been associated with a number of health risks including cancer at various sites, infections, mortality in severe illness, autoimmune thyroiditis and other diseases ${ }^{(3)}$.

Se status is mainly controlled by hepatic Se metabolism, which becomes impaired in response to cirrhosis $^{(4)}$, inflammation ${ }^{(5)}$ or hypoxia $^{(6)}$, causing diminished SePP biosynthesis and secretion. In turn, the Se concentration in blood may decline strongly. Serum Se has thus been characterised as a negative acute-phase reactant ${ }^{(7)}$. Under such conditions, the central nervous system, endocrine glands and bone may become Se-deficient and highly sensitive to oxidative injury, as SePP is the major transport protein of Se supplying these organs with the essential trace element. Collectively, the negative acute-phase response of Se together with an increased susceptibility of Se-deficient subjects for infections may form a vicious cycle of disease-dependent Se decline and impaired defence mechanisms. For these reasons, Se supplementation is considered as a potentially meaningful adjuvant treatment option in severe diseases ${ }^{(8,9)}$.

Clinically, Se status correlates with survival odds and regeneration in adult patients ${ }^{(10-12)}$. Similarly, in children, high plasma Se was associated with improved outcome in systemic inflammation $^{(13,14)}$, and a respective Cochrane meta-analysis highlighted a reduction in sepsis incidence upon Se supplementation in preterm neonates ${ }^{(15)}$. These findings are of high importance, as the immature immune system of neonates places them at increased risk for infections. High mortality rates from sepsis and pneumonia are observed in this vulnerable period, especially in poorly developed countries ${ }^{(16-18)}$. Markers of

Abbreviations: CRP, C-reactive protein; IQR, interquartile range; SePP, selenoprotein P.

* Corresponding author: L. Schomburg, fax +49 30450 524922, email: lutz.schomburg@charite.de

$\dagger$ These authors contributed equally to this work. 
oxidative stress are elevated in newborns with sepsis ${ }^{(19)}$, especially in preterm neonates ${ }^{(20)}$. These findings may be related to the Se status, as it controls the biosynthesis of selenoproteins implicated in antioxidative defence reactions and immune cell functioning. In order to test the hypothesis that infection is associated with a Se deficit already during the first few days of life, we compared two well-established plasma biomarkers of Se in infected neonates and controls. We detected some neonates with severe Se deficiency, especially in the group of preterm neonates with infection, raising the issue of Se substitution or supplementation in these very vulnerable patients.

\section{Methods \\ Study design}

The present study was designed as an observational casecontrol study. This study was conducted according to the guidelines laid down in the Declaration of Helsinki, and all procedures involving human subjects/patients were approved by the ethics committee of the Charite Universitätsmedizin Berlin, Germany (application no; EA2/092/12). Enrolment started in February 2013 and ended in April 2014 in three neonatal care units of the Charité Universitätsmedizin Berlin. Neonates fulfilling inclusion criteria (Table 1) were identified, next of kin were contacted and informed written consent for participation was obtained from one or both parents. For this study, only the remaining plasma samples from the routine diagnostic analyses were available, were pseudonymised and frozen at $-20^{\circ} \mathrm{C}$ until analysis. Leftover samples from the mothers were not available for analysis.

\section{Inclusion and exclusion criteria}

Clinical signs of infections are subtle and often non-specific in neonates ${ }^{(21,22)}$. No validated diagnosis score system for infections in early life has been established. In addition, the specificity of blood cultures in neonates is low ${ }^{(23)}$. On the basis of previous data ${ }^{(24-26)}$ and internal standard procedures, we defined an infection as the presence of at least one characteristic clinical sign and simultaneously a laboratory evidence for inflammation, as described in Table 1 . To this end, we chose IL-6 or C-reactive protein (CRP), as they are used as diagnostic markers for infections in infants and correlate with severity of infection ${ }^{(21,27,28)}$.

\section{Procedures}

Neonates with clinically suspected infections were screened for an increase in the concentration of IL- 6 and/or CRP. The first blood analysis took place immediately after detection of alarming clinical signs, in case of early-onset infection on the day of birth (day 1). Antibiotic (ampicillin and gentamicin) therapy was initiated if the IL-6 or CRP concentrations exceeded a predefined threshold (IL-6>100 ng/l; CRP >10 mg/l). To evaluate the effect of the therapy and control gentamicin blood levels, a second sample was collected after $48 \mathrm{~h}$ (day 3). These two time points were available for analysis from our infected newborns, as the study protocol allowed Se status analysis from leftover samples only. Control plasma samples were available from neonates admitted to the neonatal care units at the time of birth for diagnostic purposes unrelated to acute infections. Severe infection was defined as IL- 6 concentrations exceeding a threshold of $500 \mathrm{ng} / \mathrm{l}^{(29)}$.

\section{Subjects}

In total, 108 newborns were considered on the basis of clinical presentation, of which seventy-two fulfilled all inclusion criteria including the availability of remaining plasma samples exceeding $20 \mu \mathrm{l}$. Informed consent was obtained from forty-four parents; twenty-three from the control group and twenty-one from the infected neonates group.

\section{Measurements}

IL-6 and CRP concentrations were determined by the local commercial diagnostics provider conducting the routine clinical measurements (Labor Berlin - Charité Vivantes GmbH). CRP was measured by a turbidometric assay and IL- 6 by an electro-chemical luminescence immunoassay (COBAS 6000 or COBAS 8000; Roche Diagnostics), respectively. Se contents were determined by total reflection X-ray fluorescence analysis (S2 Picofox; Bruker Nano GmbH) as described earlier ${ }^{(30)}$. In brief, $10 \mu \mathrm{l}$ plasma samples were spiked with an internal gallium standard (Sigma-Aldrich). Samples were applied to polished quartz glass carriers, dried at $37^{\circ} \mathrm{C}$ for $3 \mathrm{~h}$ and analysed in duplicates. Quantification of SePP was carried out by analysing duplicate plasma samples using a colorimetric enzyme immunoassay (Selenotest ${ }^{\circledR}$; ICI immunochemical intelligence $\mathrm{GmbH})$ as described earlier ${ }^{(30)}$. Inter-assay and intra-assay $\mathrm{CV}$ were below $15 \%$ as determined using a commercial human reference serum sample (Seronorm; SERO AS).

\section{Western blot analysis}

Plasma samples $(0.2 \mu \mathrm{l})$ were diluted in ultrapure water (Biochrom AG) and $4 \times$ sample buffer (200 mm-Tris-HCl, $\mathrm{pH}$ 7.5; $50 \%$ glycerin; 4\% SDS; $0.04 \%$ bromophenol blue and $125 \mathrm{~mm}$-dithiothreitol). Samples were size fractionated by SDS-PAGE and blotted by semi-dry transfer onto nitrocellulose membranes (Optitran; Schleicher \& Schuell). A SePP-specific antibody (1:2000 dilution; ICI) was used, and signals were developed using the Western-Bright Quantum chemiluminescence substrate Sirius (Biozym Scientific) with the Fluor Chem FC2 detection system (Biozym).

\section{Statistics}

Statistical Package for the Social Sciences (SPSS, version 21; IBM) and GraphPad Prism (version 5; GraphPad Software, Inc.) were used for biostatistical analyses. For independent and normally distributed data, $t$ test to compare means and Pearson's test for correlation analyses were conducted. For independent and not-normally distributed variables, the Mann-Whitney $U$ and Spearman's rank correlation tests 
were used. For dependent and normally distributed variables, a paired $t$ test was used. Probability values $(P)$ of $<0.05$ were defined as significant. Univariate analyses were conducted to examine the effect of several factors on one variable. Before we conducted a univariate analysis of SePP, we performed a square root transformation to gain normally distributed groups $\left(\mathrm{SePP}_{\mathrm{sqr}}\right)$. The Se concentration of one neonate from the infected group was three times the interquartile range (IQR) above the $75 \%$ percentile $(69.26 \mu \mathrm{g} / \mathrm{l})$, and was consequently excluded from the Se analysis.

\section{Results}

A total of forty-four subjects fulfilled all inclusion criteria (Table 1) and were included in our study, of which twenty-three were classified as control and twenty-one as neonates with infection. The other samples initially collected were not analysed, as the sample volume was insufficient or consent from the parent(s) was not available (denied or incomplete, $n$ 28). Table 2 presents the anthropometric and clinical characteristics of the neonates finally enrolled in the study. Overall, neonates in the control group had on average a lower gestational age and a lower birth weight. Sex distribution differed between the groups with more female newborns in the control group than in the group with connatal infection (Table 2).
The average Se concentration on day 0 of the newborns in our study was 34.5 (sD 11.6) $\mu \mathrm{g} / \mathrm{l}$, considerably below the levels usually observed in adults. Se and SePP concentrations were strongly correlated $\left(b=0.025, r_{p}=0.78, P<0.001\right)$, verifying the notion that Se status was suboptimal in the newborns. The positive correlation was present both in the control group (Fig. 1(a)) and in the group of infected newborns (Fig. 1(b)). When comparing the infected neonates before (day 1) and after (day 3) initiation of antibiotic therapy, the correlation was stronger at day 1 compared with day 3 (Fig. 1(c) and (d)).

On average and without considering covariates, mean plasma Se concentrations of the infected infants were lower compared with the control group at day 1 , but not significantly different (Fig. 2(a)). Very low Se concentrations $(\mathrm{Se}<20 \mu \mathrm{g} / \mathrm{l})$ were observed in four infected subjects $(11 \cdot 7,14 \cdot 3,15 \cdot 1$ and $15.7 \mu \mathrm{g} / \mathrm{l})$ and one control subject $(15.8 \mu \mathrm{g} / \mathrm{l})$. Similarly, mean SePP concentrations in our study were not significantly different between control and infected newborns (Fig. 2(b)). Notably, the two biomarkers of Se status showed a relatively wide range of concentrations both in the controls (median Se, 36.2 (IQR 10.8) $\mu \mathrm{g} / \mathrm{l}$; median SePP, 0.99 (IQR 0.50) $\mathrm{mg} / \mathrm{l}$ ) and in the infected newborns (Se, 31.8 (IQR 10.5) $\mu \mathrm{g} / \mathrm{l}$; SePP, 0.98 (IQR 0.53) mg/l)).

Gestational age was not correlated with Se or SePP concentrations in the group of controls (Fig. 3(a) and (c)). However, both biomarkers of Se status were positively correlated with gestational age in the group of infected newborns (Fig. 3(b) and (d)).

Table 1. Inclusion and exclusion criteria

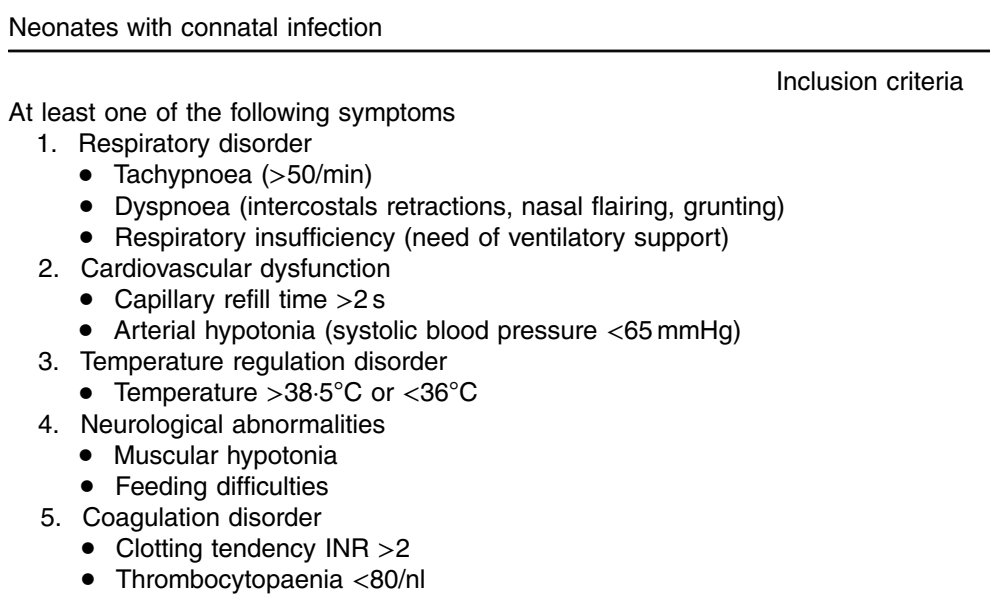

Inclusion criteria

In combination with laboratory evidence for inflammation

- IL-6 $>100 \mathrm{ng} / \mathrm{l}$ or CRP $>10 \mathrm{mg} / \mathrm{l}$ on day of birth

Antibiotic treatment for at least $3 d$

Second sample $2 \mathrm{~d}$ after diagnosis (routine control of gentamicin plasma concentrations)

$\geq 20 \mu \mathrm{l}$ residual plasma from both day 1 and day 3

Inborn neonate and admission to the neonatal unit

\title{
Controls
}

\author{
)
}

(20) 
Table 2. Group characteristics

(Medians and interquartile ranges (IQR) and standard deviations)

\begin{tabular}{|c|c|c|c|c|c|}
\hline \multirow[b]{2}{*}{ Parameters } & \multicolumn{2}{|c|}{ Infection group ( $n$ 21) } & \multicolumn{2}{|c|}{ Control group (n 23) } & \multirow[b]{2}{*}{$P$} \\
\hline & Median & IQR & Value & IQR & \\
\hline Male & \multirow{2}{*}{\multicolumn{2}{|c|}{$n 15$}} & \multicolumn{2}{|c|}{$n 8$} & - \\
\hline Female & & & \multicolumn{2}{|c|}{$n 15$} & - \\
\hline Gestational age (weeks) & 38.4 & $35 \cdot 1-39 \cdot 8$ & 34.9 & $33 \cdot 9-37 \cdot 1$ & $0.003^{*}$ \\
\hline Weight at birth (g) & & & & & $0.003^{*}$ \\
\hline Median & \multirow{2}{*}{\multicolumn{2}{|c|}{3120}} & \multirow{2}{*}{\multicolumn{2}{|c|}{2452}} & \\
\hline SD & 734 & & & & \\
\hline Apgar (1 min) & 8.0 & $4 \cdot 5-9 \cdot 0$ & $9 \cdot 0$ & $6 \cdot 0-9 \cdot 0$ & 0.505 \\
\hline Apgar (5 min) & $9 \cdot 0$ & $7 \cdot 5-10 \cdot 0$ & $9 \cdot 0$ & $8 \cdot 0-10 \cdot 0$ & 0.951 \\
\hline IL-6 (pg/ml) on day 1 & $498 \cdot 5$ & $203 \cdot 6-2523 \cdot 0$ & $5 \cdot 8$ & $4 \cdot 2-17 \cdot 0$ & $<0.001^{*}$ \\
\hline $\mathrm{CRP}(\mathrm{mg} / \mathrm{l})$ on day 1 & 4.9 & $0.8-26 \cdot 7$ & - & - & - \\
\hline $\mathrm{CRP}(\mathrm{mg} / \mathrm{l})$ on day 3 & $12 \cdot 8$ & $5 \cdot 0-18.9$ & - & - & - \\
\hline $\mathrm{pH}$ (cord-blood) & $7 \cdot 23$ & $7 \cdot 19-7 \cdot 3$ & $7 \cdot 25$ & $7.20-7.29$ & 0.655 \\
\hline $\mathrm{pH}$ on admission & $7 \cdot 33$ & $7 \cdot 26-7 \cdot 37$ & $7 \cdot 33$ & $7 \cdot 27-7 \cdot 38$ & 0.673 \\
\hline Base excess on admission & $-2 \cdot 80$ & -5.32 to -1.28 & $-2 \cdot 15$ & -3.75 to -0.90 & 0.283 \\
\hline Twins & \multicolumn{2}{|c|}{$n 1$} & \multicolumn{2}{|c|}{$n 7$} & - \\
\hline Vaginal birth & \multicolumn{2}{|c|}{$n 10$} & \multicolumn{2}{|c|}{$n 7$} & - \\
\hline Duration of antibiotics (d) & $5 \cdot 0$ & $3.0-5 \cdot 0$ & - & - & - \\
\hline
\end{tabular}

CRP, C-reactive protein.

* Significant difference $(P<0.05)$.

(a)

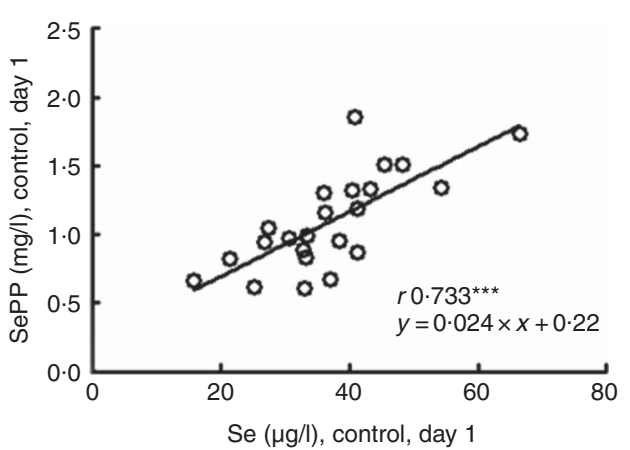

(c)

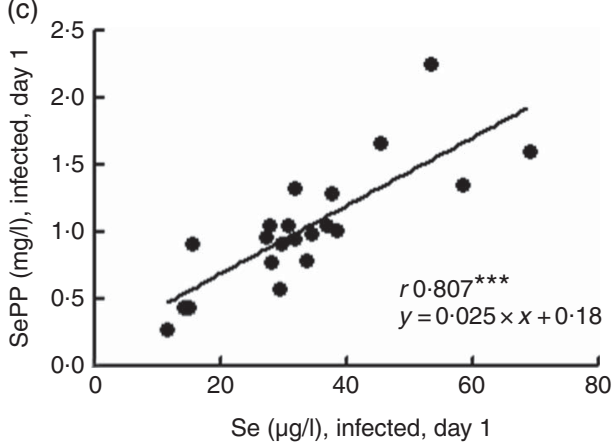

(b)
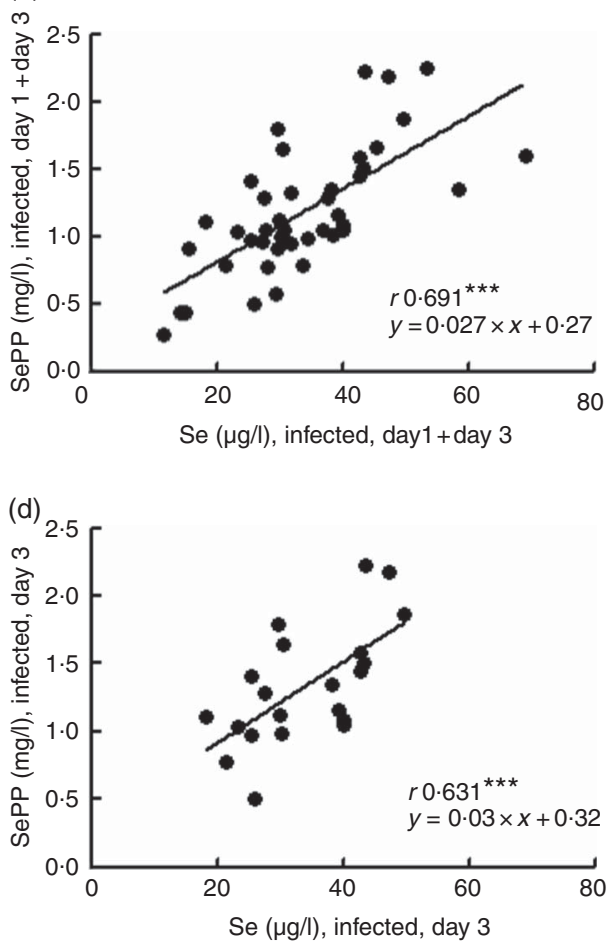

Fig. 1. Comparison of the two selenium biomarkers analysed in this study. Total selenium and selenoprotein P (SePP) show a strong and positive correlation in (a) the control and (b) in the group of infected newborns. The strong correlation of selenium and SePP is observed in the infected newborns both at (c) birth and (d) $48 \mathrm{~h}$ after initiation of treatment. These data indicate that both total selenium and SePP concentrations are suitable for Se status assessment in healthy and infected newborns. ${ }^{\star \star \star} P<0.001$.

In particular, preterm infants (gestational age $<37$ weeks) had lower SePP concentrations (median SePP, 0.91 (IQR 0.51) mg/l) than term infants (SePP, 1.04 (IQR 0.57) mg/l). This difference was statistically significant $(U=145, \quad z=-2 \cdot 192, \quad P=0 \cdot 028)$. In comparison, Se concentrations were not significantly different between preterm and term infants (median (preterm) $=32.6 \mu \mathrm{g} / \mathrm{l} ; v$. median $($ term $)=39 \cdot 0 \mu \mathrm{g} / 1 ; U=175, z=-1.481, P=0 \cdot 139)$.
Next, we conducted a univariate analysis with gestational age as a covariate and infection status as a fixed factor to examine their influence on Se and SePP concentrations. No previous studies have created evidence for a positive correlation between infection risk and gestational age, and therefore the difference in age between the groups seems to be a random error (Table 2$)^{(31)}$. In this analysis, gestational age was 
(a)

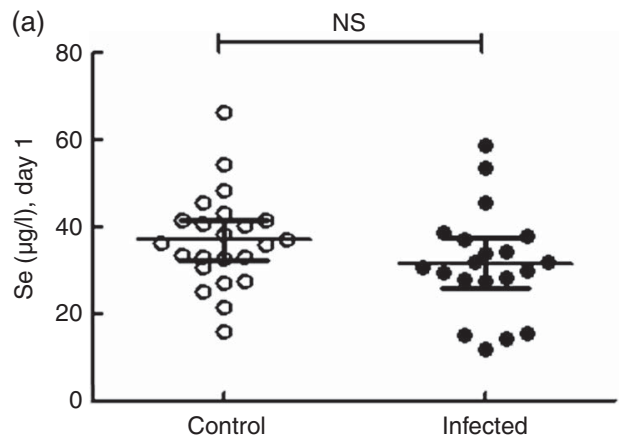

(b)

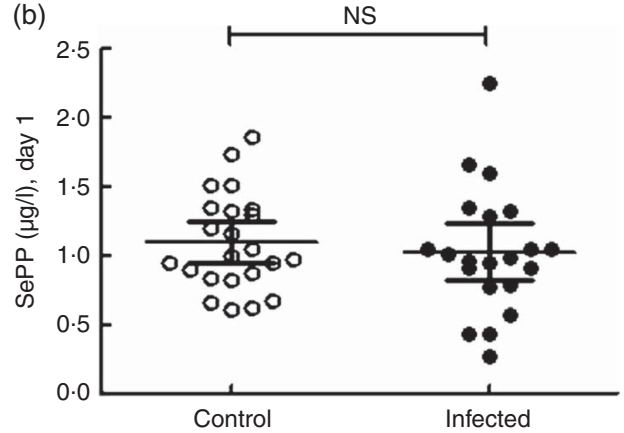

Fig. 2. Plasma concentrations of selenium and selenoprotein $P$ (SePP) in control and infected newborns. (a) The selenium concentrations in both groups cover a relatively wide range and are on average not different between controls and infected neonates. (b) The ranges of SePP concentrations are similarly wide in both groups and do not differ on average between control and infected. Notably, the lowest selenium and SePP concentrations are detected in the group of infected newborns.
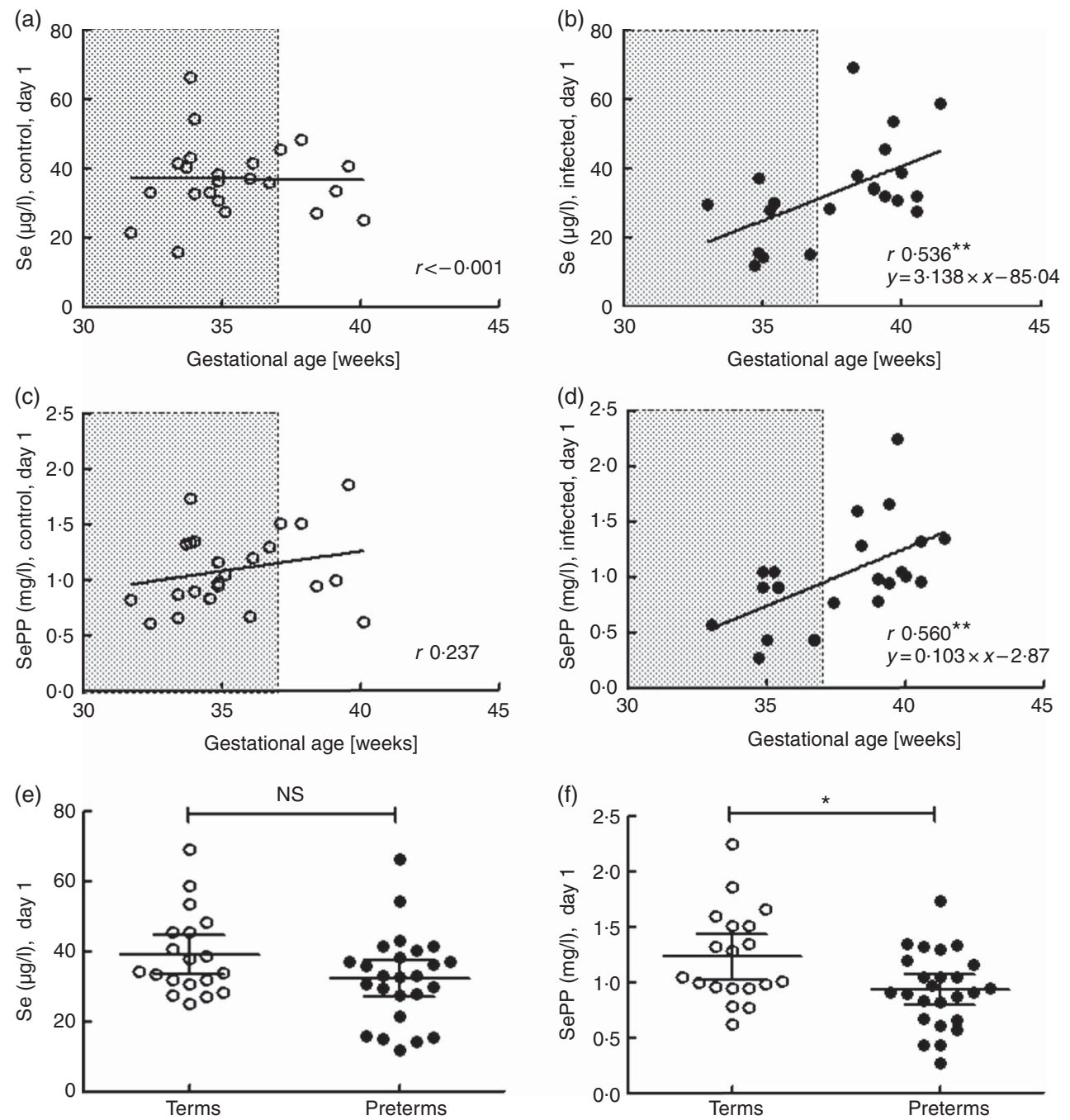

Fig. 3. Association of selenium status with gestational age. (a, c) Concentrations of selenium and selenoprotein $P$ (SePP) were unrelated to gestational age in the neonates of the control group. (b, d) In the infected neonates, selenium and SePP concentrations were positively associated with gestational age; (b) $P=0.006$, and (d) $P=0.004$. Notably, the lowest levels of selenium and SePP observed in this study were detected in premature neonates with infection. (e, f) Separating all newborns into term ( $>37$ weeks) and preterm, there was no significant difference in (e) selenium concentrations, whereas (f) SePP concentrations differed significantly. 圈, Preterms. ${ }^{*} P<0.05$, ${ }^{\star *} P<0.01$.

significantly related to Se concentration $(P=0 \cdot 045, b=1 \cdot 48$, 95\% CI 0.038, 2.923). After controlling for gestational age, infection had a significant effect on concentrations of Se
$(P=0.027, b=-8.584,95 \% \mathrm{CI}-16 \cdot 143,-1.026)$ and SePP ${ }_{\text {sqrt }}$ ( $P=0.045, b=-0 \cdot 125,95 \% \mathrm{CI}-0 \cdot 247,-0.003)$. The univariate analysis with birth weight as a covariate and infection status as a 
fixed factor yielded a similarly strong significant relation to Se concentrations ( $P=0.031, b=-8.381,95 \% \mathrm{CI}-0.796,-15.966$ ), but failed to reach statistical significance for SePP sqrt concentrations $(P=0 \cdot 087, b=-0 \cdot 109,95 \% \mathrm{CI}-0 \cdot 235,0 \cdot 017)$.

With respect to effects of therapy, Se concentrations were not significantly different between day of birth (day 1) and $48 \mathrm{~h}$ after initiation of treatment (day 3) (Fig. 4(a)). However, SePP concentrations increased significantly from day 1 to day 3 (Fig. 4(b)). There was a significant negative correlation between gentamicin and Se concentrations on day 3 (Fig. 4(c)). Western blot analysis was performed in order to verify the data obtained from ELISA and to test for differently migrating SePP isoforms (Fig. 4(e)). Measured SePP concentrations were congruent with the optical density of the SePP-specific Western blot bands, and no indications for additional SePP isoforms were noted.

The influence of infection on Se status was evident in the early stage (Fig. 5(a) and (b)). IL-6 levels were inversely correlated to both Se and SePP on day 1 in neonates with severe infection (IL-6 $>500 \mathrm{ng} / \mathrm{l}$ ), whereas on day 3 there was no correlation between Se or SePP with CRP concentrations.

\section{Discussion}

Se deficiency is associated with increased mortality in adults in intensive care ${ }^{(10,11,32)}$. Whether the same association holds in neonates is unknown. We decided to assess the Se status of newborns with and without infection as a first step towards a better understanding of this potential interaction in the first few days of life. We hypothesised that infection negatively affects hepatic selenoprotein biosynthesis as shown before in model systems $^{(5,33)}$. Therefore, we aimed to determine the two important biomarkers of Se status - that is, total plasma Se and SePP concentrations.

The strong correlation observed between these two biomarkers indicates that both parameters are suitable for assessing Se status in neonates. It is well established that
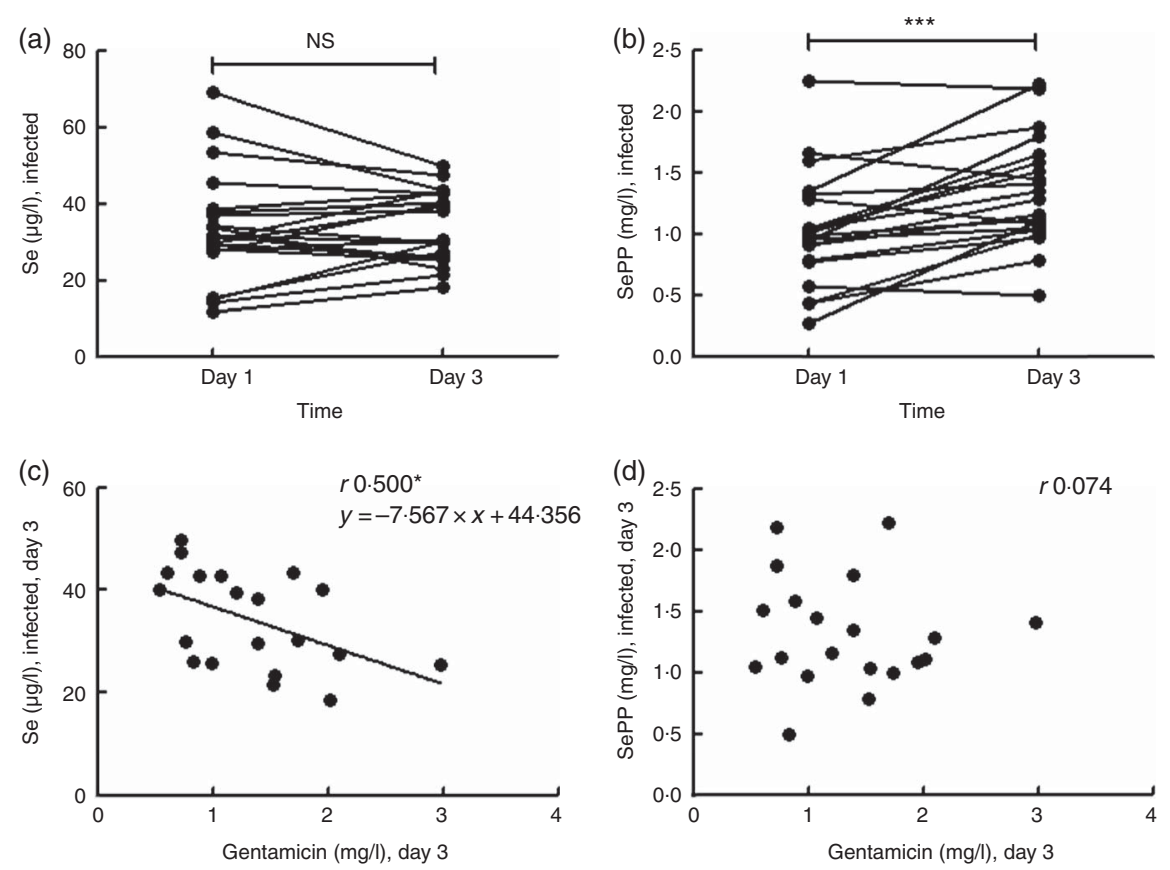

(e)

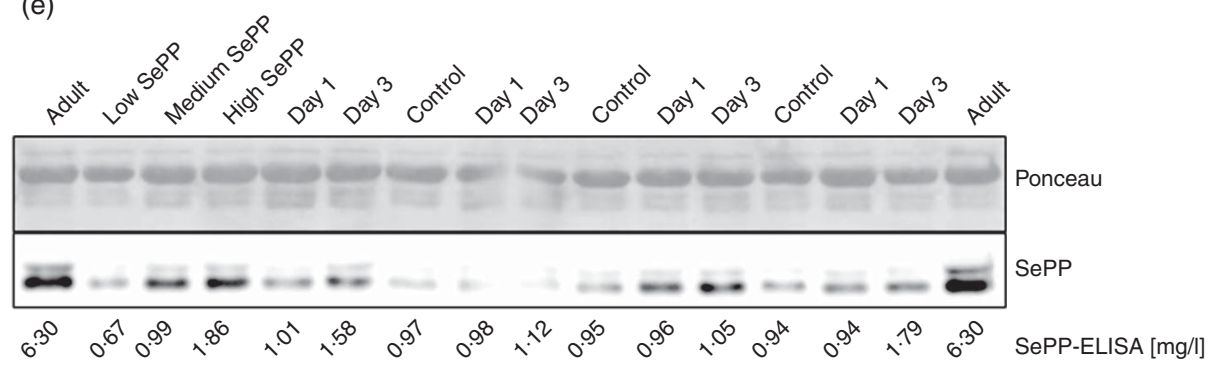

Fig. 4. Analysis of selenium and selenoprotein $P$ (SePP) concentrations in infected neonates on day 1 and day 3 of treatment. (a) Average selenium concentrations do not differ between the two time points. The relatively wide range of selenium concentrations observed on day 1 is diminished on day 3 . (b) SePP is significantly $(P<0.001)$ elevated in the samples from the treated newborns (day 3 ) as compared with treatment initiation (day 1). (c) The blood concentration of gentamicin correlates negatively with selenium concentrations on day 3 (d) In parallel, no such correlation is observed between SePP and gentamicin. (e) Immunoreactive SePP band density (SePP) was in agreement with the concentrations determined by ELISA (SePP-ELISA), and no indication for a shift in SePP isoform pattern was observed. The Western blot analysis of four infected neonates along with standards (low, medium and high SePP) and controls (control and an adult sample) is shown. ${ }^{*} P<0.05,{ }^{\star \star \star} P<0.001$. 
(a)
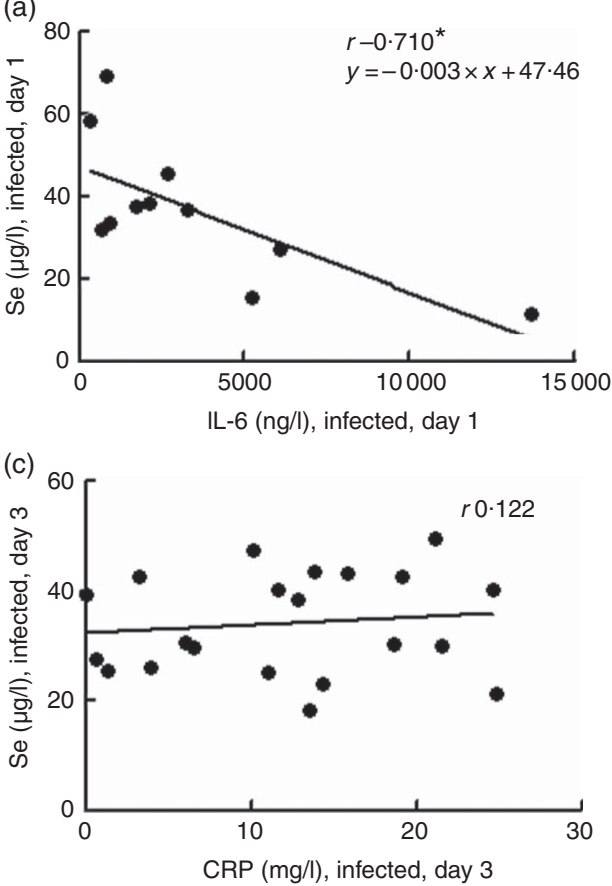

(b)
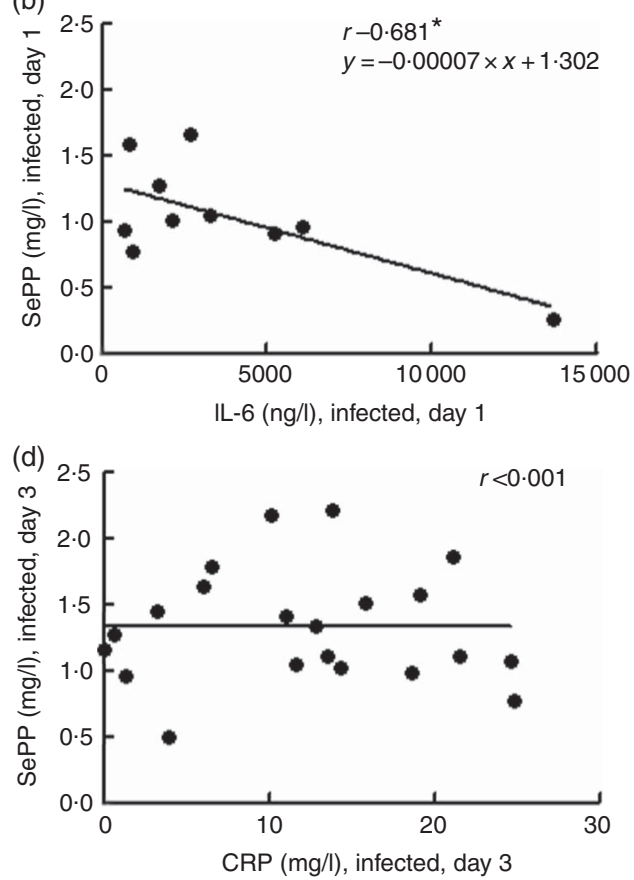

Fig. 5. Association of severe infection with biomarkers of selenium status. IL-6 is negatively associated with (a) selenium and (b) selenoprotein $P$ (SePP) at birth (day 1 ) in neonates with severe infection (IL-6 $>500 \mathrm{ng} / \mathrm{l})$. On day $3(48 \mathrm{~h}$ after initiation of therapy), C-reactive protein (CRP) concentrations were neither associated with (c) selenium nor with (d) SePP. * $P<0.05$.

selenoprotein expression becomes saturated at sufficiently high Se intake, causing a disruption of the strong positive correlation between Se and SePP ${ }^{(34)}$. Consequently, our data indicate that the majority of neonates in our study were not sufficiently well supplied with Se for maximal selenoprotein expression. This is further substantiated by comparing the absolute concentrations in relation to adults; average Se and SePP concentrations of the neonates in our study at day 1 were $35 \cdot 2$ (SD 12.6) $\mu \mathrm{g} \mathrm{Se/1}$ and $1 \cdot 1$ (SD 0.4) $\mathrm{mg} \mathrm{SePP} / 1$, respectively, which corresponds to only 40 and $25 \%$ of the concentrations found in adults, respectively, as, for example, determined in a recent large study of colorectal cancer risk in Europe ${ }^{(30)}$. Even German vegetarians, who are at high risk of Se deficiency, had about 2-fold higher Se concentrations as compared with the neonates in this study ${ }^{(35)}$. Similarly, the reference value determined in Swiss and German infants $<1$ month of age lies at $0.64 \mu \mathrm{m}-\mathrm{Se}$ (corresponding to $50.6 \mu \mathrm{g} / \mathrm{l}$ ), again 1.4 times above the average of the neonates in our study ${ }^{(37)}$.

Thus, the relatively low average plasma Se concentrations determined in both groups of neonates may reflect that many of the enrolled infants were very premature and in a postnatal phase in which Se status may still increase continuously with each day of life. Moreover, we observed that preterm infants have significantly lower SePP levels than term infants. This notion is in agreement with similar findings of Se concentrations in other studies ${ }^{(36-38)}$. The underlying reason for higher plasma SePP in term as compared with preterm neonates is unknown, but may be related to the maturation of the hepatic selenoprotein biosynthesis machinery during development, to an increasing generation of a hepatic Se store until delivery or to changes in intestinal absorption and chorionic villi function as hypothesised in a recent meta-analysis on Se deficiency in preterm infants ${ }^{(39)}$.

The univariate analysis with gestational age as a covariate revealed a significant effect of an infection on both Se and SePP concentrations, supporting the hypothesis that the Se status of neonates declines during infection. Whether a reduced Se status has a negative impact on the severity, duration of stay in the intensive care unit or treatment success of neonatal infection needs to be examined in future studies.

As we were not able to analyse blood from healthy neonates, for ethical reasons, we determined whether there is an association of clinical markers of infection with Se status. An inverse relationship was observed when comparing Se and SePP levels with IL-6 concentrations (Fig. 5(a) and (b)). In particular, two infants with very high levels of IL- 6 at birth $(13690,5247 \mathrm{pg} / \mathrm{ml})$ displayed very low concentrations of Se $(11.7,15.7 \mu \mathrm{g} / \mathrm{l})$ and $\operatorname{SePP}(0.27,0.91 \mathrm{mg} / \mathrm{l})$. This finding is in agreement with our recent analysis on the impact and importance of IL-6 for hepatic SePP biosynthesis and secretion ${ }^{(40)}$.

Previous studies have shown that both biomarkers - that is, total Se and SePP - are suitable to determine a Se-deficient state, whereas only total Se assessment appears suitable to detect Se intoxication $^{(41)}$. The positive and almost linear correlation of both biomarkers observed in countries with marginal intake suggests that it may make no difference if measuring one or the other in order to assess Se status. In this respect, neonates were already showing the same strong association as known from healthy or diseased adults in Se-deficient populations ${ }^{(42)}$. Yet, our two recent large-scale studies on the influence of Se status on bone turnover in healthy postmenopausal women ${ }^{(43)}$ and on 
colorectal cancer risk in European adults ${ }^{(30)}$ both indicated that the interactions of Se status and clinical parameters are in general stronger for SePP than for total Se. This may be because of the role of SePP as the major determinant of plasma Se concentrations and its transport function in blood, being specifically recognised and taken up by receptors on target cells as the peripherally bioavailable fraction of Se in blood ${ }^{(44,45)}$.

In addition to the differences between the control and infection groups, we found a significant difference between day 1 and day 3 in SePP concentrations. The antibiotic treatment successfully corrected the severe Se and SePP deficiency of the newborns with highest IL-6 concentrations and lowest Se status. During this time period, SePP concentrations increased significantly, whereas total Se concentrations remained unchanged. This difference may indicate an improved rate of SePP biosynthesis in hepatocytes and a more efficient anabolic usage of circulating Se forms during therapy, again highlighting SePP as the probably more suitable and sensitive biomarker under these conditions. The biosynthesis of Se-poor SePP isoforms terminating before the Se-rich protein domain may provide an alternative explanation ${ }^{(46)}$. Similarly, a more efficient selenoprotein biosynthesis read-through due to the effects of the aminoglycoside treatment on ribosomal amino acid insertion may have caused increasing SePP concentrations during therapy, as described before in in vitro studies for selenoproteins glutathione peroxidase $1^{(47)}$ and $\operatorname{SePP}^{(6)}$. Notably, under aminoglycoside exposure, the error rate of UGA (stop-codon) decoding increases strongly, giving rise to inactive and Se-deprived selenoprotein variants, as convincingly shown in breast cancer cells by Tobe et al. ${ }^{(48)}$ using a combination of radioactive labelling, protein and activity analyses as well as MS.

This hypothesis is supported by our data on the relation of Se and SePP after gentamicin treatment. If gentamicin causes increased read-through at UGA stop codons, yielding Se-poor SePP variants as a consequence of amino acid mis-incorporation, then plasma Se concentrations should negatively correlate with the gentamicin level (Fig. 4(c)), without leading to a similar effect on plasma SePP concentrations, which may under such conditions reflect both full-length Se-rich and Se-poor SePP variants (Fig. 4(d)). This hypothesis should be tested in future studies with respect to the Se content of circulating SePP, which will likely be only possible with adult patients under aminoglycoside therapy, because of the high demand for serum or plasma volume. Under these conditions, we found no indications in our Western blot analyses that different or shorter SePP isoforms were generated. The amount of full-size SePP increased in most of the neonates from day 1 to day 3 in agreement with ELISA results. This increase may be due to an improved control of the inflammatory cytokines during therapy, and may have resulted from the abovementioned stimulation of selenoprotein biosynthesis by the aminoglycosides. Alternatively, or in addition to the effects described, the antibiotic may have affected the composition of the intestinal microflora, which has been shown in a mouse model to affect Se availability to the host and hepatic selenoprotein biosynthesis ${ }^{(49)}$. From a therapeutic point of view, the bioavailabilities of SePP being synthesised in the presence or absence of aminoglycosides need to be compared, in order to determine whether Se transport becomes impaired under gentamicin therapy.
The low sample requirement in combination with the ease and speed of immunological detection may favour SePP assessment over total Se determination as the preferred method of analysis in infected subjects in future studies. Especially in neonates, only minute amounts of plasma are usually available and highly sensitive techniques are needed. If a fast and reliable bedside test for SePP quantification could be established, it would allow the identification of severely Se-deficient neonates already during intensive care unit admission and a fast initiation of measures for correcting a potential Se deficit. Luckily, all neonates included in our study survived the connatal infection. Nevertheless, a severe Se deficiency needs to be considered as a relevant mortality risk factor, and Se supplementation may have beneficial effects in severely diseased neonates as documented in some but not all respective studies with adult patients ${ }^{(13,50-53)}$. Alternatively, the Se status of the mothers may be adjusted for avoiding Se deficiency during pregnancy. Such measures would not only be of likely advantage to the unborn child but may also reduce the risks of adverse pregnancy outcomes ${ }^{(54)}$. Therefore, this direction of research needs to be continued, as reducing child mortality is among the millennium goals ${ }^{(55)}$. We hope that our study contributes to this challenge and supports the initiation of more focused studies on the health importance of Se in connatal infections for the sake of the most vulnerable patients.

\section{Acknowledgements}

The authors express the gratitude to all parents and children who took part in this study, and to the clinical colleagues taking care of the patients in the Department of Neonatology, Charité Universitätsmedizin Berlin, Campus Virchow-Klinikum, Germany.

This work was supported by Deutsche Forschungsgemeinschaft (DFG) grants RE3038/1-1 (to K. R.) and Scho849/4-1 (to L. S.), and an Elsa-Neumann stipend from the City of Berlin (to J. M.).

L. W., M. C., M. W., N.-P. B., C. B. and L. S. formulated the research question and designed the study. L. W., M. W., N.-P. B., E. R., J. M., S. H. and K. R. conducted the experimental analyses; all authors contributed to the statistical analysis and interpretation of the data. All authors contributed to writing and editing the manuscript.

None of the authors has any conflicts of interest to disclose.

\section{References}

1. Labunskyy VM, Hatfield DL \& Gladyshev VN (2014) Selenoproteins: molecular pathways and physiological roles. Physiol Rev 94, 739-777.

2. Schweizer U, Dehina N \& Schomburg L (2011) Disorders of selenium metabolism and selenoprotein function. Curr Opin Pediatr 23, 429-435.

3. Rayman MP (2012) Selenium and human health. Lancet 379, 1256-1268.

4. Burk RF, Early DS, Hill KE, et al. (1998) Plasma selenium in patients with cirrhosis. Hepatology 27, 794-798.

5. Renko K, Hofmann PJ, Stoedter M, et al. (2009) Downregulation of the hepatic selenoprotein biosynthesis machinery impairs selenium metabolism during the acute phase response in mice. FASEB J 23, 1758-1765. 
6. Becker NP, Martitz J, Renko K, et al. (2014) Hypoxia reduces and redirects selenoprotein biosynthesis. Metallomics 6 , 1079-1086.

7. Nichol C, Herdman J, Sattar N, et al. (1998) Changes in the concentrations of plasma selenium and selenoproteins after minor elective surgery: further evidence for a negative acute phase response? Clin Chem 44, 1764-1766.

8. Heyland DK (2007) Selenium supplementation in critically ill patients: can too much of a good thing be a bad thing? Crit Care 11, 153.

9. Schomburg L (2014) Selenium in sepsis - substitution, supplementation or pro-oxidative bolus? Crit care 18, 444.

10. Forceville X, Vitoux D, Gauzit R, et al. (1998) Selenium, systemic immune response syndrome, sepsis, and outcome in critically ill patients. Crit Care Med 26, 1536-1544.

11. Angstwurm MW, Engelmann L, Zimmermann T, et al. (2007) Selenium in Intensive Care (SIC): results of a prospective randomized, placebo-controlled, multiple-center study in patients with severe systemic inflammatory response syndrome, sepsis, and septic shock. Crit Care Med 35, 118-126.

12. Meyer HA, Endermann T, Stephan C, et al. (2012) Selenoprotein P status correlates to cancer-specific mortality in renal cancer patients. PLOS ONE 7, e46644.

13. Leite HP, Nogueira PC, de Oliveira Iglesias SB, et al. (2015) Increased plasma selenium is associated with better outcomes in children with systemic inflammation. Nutrition 31, 485-490.

14. Iglesias SB, Leite HP, Paes AT, et al. (2014) Low plasma selenium concentrations in critically ill children: the interaction effect between inflammation and selenium deficiency. Crit Care 18, R101.

15. Darlow BA \& Austin NC (2003) Selenium supplementation to prevent short-term morbidity in preterm neonates. The Cochrane Database of Systematic Reviews, 2003, Issue 4. Art. No.: CD003312.

16. World Health Organization (2014) World Health Statistics 2014. Geneva: WHO.

17. Molyneux E \& Gest A (2015) Neonatal sepsis: an old issue needing new answers. Lancet Infect Dis 15, 503-505.

18. Seale AC, Blencowe H, Manu AA, et al. (2014) Estimates of possible severe bacterial infection in neonates in sub-Saharan Africa, south Asia, and Latin America for 2012: a systematic review and meta-analysis. Lancet Infect Dis 14, 731-741.

19. Cancelier AC, Petronilho F, Reinke A, et al. (2009) Inflammatory and oxidative parameters in cord blood as diagnostic of early-onset neonatal sepsis: a case-control study. Pediatr Crit Care Med 10, 467-471.

20. Buonocore G, Perrone S, Longini M, et al. (2002) Oxidative stress in preterm neonates at birth and on the seventh day of life. Pediatr Res 52, 46-49.

21. Arnon S \& Litmanovitz I (2008) Diagnostic tests in neonatal sepsis. Curr Opin Infect Dis 21, 223-227.

22. Shah BA \& Padbury JF (2014) Neonatal sepsis: an old problem with new insights. Virulence 5, 170-178.

23. Vergnano S, Menson E, Kennea N, et al. (2011) Neonatal infections in England: the NeonIN surveillance network. Arch Dis Child Fetal Neonat Ed 96, F9-F14.

24. Young Infants Clinical Signs Study Group (2008) Clinical signs that predict severe illness in children under age 2 months: a multicentre study. Lancet 371, 135-142.

25. Goldstein B, Giroir B, Randolph A, et al. (2005) International pediatric sepsis consensus conference: definitions for sepsis and organ dysfunction in pediatrics. Pediatr Crit Care Med 6, 2-8.

26. Dollner H, Vatten L \& Austgulen R (2001) Early diagnostic markers for neonatal sepsis: comparing C-reactive protein, interleukin-6, soluble tumour necrosis factor receptors and soluble adhesion molecules. J Clin Epidemiol 54, 1251-1257.
27. Chiesa C, Pacifico L, Natale F, et al. (2015) Fetal and early neonatal interleukin-6 response. Cytokine 76, 1-12.

28. Ng PC \& Lam HS (2006) Diagnostic markers for neonatal sepsis. Curr Opin Pediatr 18, 125-131.

29. Bloos F \& Reinhart K (2014) Rapid diagnosis of sepsis. Virulence 5, 154-160.

30. Hughes DJ, Fedirko V, Jenab M, et al. (2015) Selenium status is associated with colorectal cancer risk in the European prospective investigation of cancer and nutrition cohort. Int J Cancer 136, 1149-1161.

31. Miller GA \& Chapman JP (2001) Misunderstanding analysis of covariance. J Abnorm Psychol 110, 40-48.

32. Sakr Y, Reinhart K, Bloos F, et al. (2007) Time course and relationship between plasma selenium concentrations, systemic inflammatory response, sepsis, and multiorgan failure. Br J Anaesth 98, 775-784.

33. Wang Z, Forceville X, Van Antwerpen P, et al. (2009) A largebolus injection, but not continuous infusion of sodium selenite improves outcome in peritonitis. Shock 32, 140-146.

34. Hurst R, Armah CN, Dainty JR, et al. (2010) Establishing optimal selenium status: results of a randomized, double-blind, placebo-controlled trial. Am J Clin Nutr 91, 923-931.

35. Hoeflich J, Hollenbach B, Behrends T, et al. (2010) The choice of biomarkers determines the selenium status in young German vegans and vegetarians. Br J Nutr 104, 1601-1604.

36. Iranpour R, Zandian A, Mohammadizadeh M, et al. (2009) Comparison of maternal and umbilical cord blood selenium levels in term and preterm infants. Zhongguo Dang Dai Er Ke Za Zbi 11, 513-516.

37. Loui A, Raab A, Braetter P, et al. (2008) Selenium status in term and preterm infants during the first months of life. Eur J Clin Nutr 62, 349-355.

38. Sievers E, Arpe T, Schleyerbach U, et al. (2001) Plasma selenium in preterm and term infants during the first 12 months of life. J Trace Elem Med Biol 14, 218-222.

39. Freitas RG, Nogueira RJ, Antonio MA, et al. (2014) Selenium deficiency and the effects of supplementation on preterm infants. Rev Paul Pediatr 32, 126-135.

40. Martitz J, Becker NP, Renko K, et al. (2015) Gene-specific regulation of hepatic selenoprotein expression by interleukin-6. Metallomics 7, 1515-1521.

41. Combs GF Jr (2015) Biomarkers of selenium status. Nutrients 7, 2209-2236.

42. Burk RF \& Hill KE (2009) Selenoprotein P-expression, functions, and roles in mammals. Biochim Biophys Acta 1790, 1441-1447.

43. Hoeg A, Gogakos A, Murphy E, et al. (2012) Bone turnover and bone mineral density are independently related to selenium status in healthy euthyroid postmenopausal women. J Clin Endocrinol Metab 97, 4061-4070.

44. Olson GE, Winfrey VP, Hill KE, et al. (2008) Megalin mediates selenoprotein $\mathrm{P}$ uptake by kidney proximal tubule epithelial cells. J Biol Chem 283, 6854-6860.

45. Olson GE, Winfrey VP, Nagdas SK, et al. (2007) Apolipoprotein E receptor-2 (ApoER2) mediates selenium uptake from selenoprotein $\mathrm{P}$ by the mouse testis. $\mathrm{J} \mathrm{Biol} \mathrm{Chem}$ 282, 12290-12297.

46. Kurokawa S, Bellinger FP, Hill KE, et al. (2014) Isoformspecific binding of selenoprotein $\mathrm{P}$ to the beta-propeller domain of apolipoprotein $\mathrm{E}$ receptor 2 mediates selenium supply. J Biol Chem 289, 9195-9207.

47. Handy DE, Hang G, Scolaro J, et al. (2006) Aminoglycosides decrease glutathione peroxidase- 1 activity by interfering with selenocysteine incorporation. J Biol Chem 281, 3382-3388.

48. Tobe R, Naranjo-Suarez S, Everley RA, et al. (2013) High error rates in selenocysteine insertion in mammalian cells treated 
with the antibiotic doxycycline, chloramphenicol, or geneticin. J Biol Chem 288, 14709-14715.

49. Kasaikina MV, Kravtsova MA, Lee BC, et al. (2011) Dietary selenium affects host selenoproteome expression by influencing the gut microbiota. FASEB J 25, 2492-2499.

50. Alhazzani W, Jacobi J, Sindi A, et al. (2013) The effect of selenium therapy on mortality in patients with sepsis syndrome: a systematic review and meta-analysis of randomized controlled trials. Crit Care Med 41, 1555-1564.

51. Manzanares W, Biestro A, Torre MH, et al. (2011) High-dose selenium reduces ventilator-associated pneumonia and illness severity in critically ill patients with systemic inflammation. Intensive Care Med 37, 1120-1127.
52. Manzanares W, Langlois PL \& Hardy G (2013) Selenium pharmaconutrition in sepsis: to give or not to give? Is this still the question? Nutrition 29, 1429-1430.

53. Kong Z, Wang F, Ji S, et al. (2013) Selenium supplementation for sepsis: a meta-analysis of randomized controlled trials. $\mathrm{Am}$ J Emerg Med 31, 1170-1175.

54. Mariath AB, Bergamaschi DP, Rondo PH, et al. (2011) The possible role of selenium status in adverse pregnancy outcomes. Br J Nutr 105, 1418-1428.

55. Lozano R, Wang H, Foreman KJ, et al. (2011) Progress towards Millennium Development Goals 4 and 5 on maternal and child mortality: an updated systematic analysis. Lancet $\mathbf{3 7 8}$, $1139-1165$. 